ISBN 82-553-0821-0

Pure Mathematics
No 1

January 1993

\title{
Asymptotics of periodic subelliptic operators
}

by

C. J.K. Batty, O. Bratteli, P. E.T. Jørgensen and D.W. Robinson

PREPRINT SERIES - Matematisk institutt, Universitetet i Oslo 


\title{
Asymptotics of periodic subelliptic operators
}

\author{
Charles J. K. Batty ${ }^{1}$, Ola Bratteli ${ }^{2}$, Palle E. T. Jørgensen ${ }^{3}$ \\ and \\ Derek W. Robinson
}

Centre for Mathematics and its Applications

School of Mathematical Sciences

Australian National University

GPO Box 4

Canberra, ACT 2601

Australia

December1992

AMS Subject Classification: 43A65, 22E45, 35H05, 22E25, 35B45.

\begin{abstract}
We establish that heat diffusion with periodic conductivity is governed by two scales. The small time diffusion is described by the geodesic distance but the large time behaviour is dictated by the distance associated with an homogenized system obtained by a suitable averaging process. Our methods are quite general and apply to diffusion on a stratified Lie group.
\end{abstract}

1. Permanent address: St. John's College, Oxford, OX1 3JP, U. K.

2. Permanent address: University of Oslo, P. B. 1053 Blindern, N-0316 Oslo 3, Norway

3. Permanent address: University of Iowa, Iowa City, IA-52242-1466 USA 


\section{Introduction}

There is now an extensive literature on Gaussian bounds for heat kernels associated with subelliptic or strongly elliptic operators (see [Dav1] [Rob] [VSC] and references therein). The best upper bounds have been derived by a technique introduced by Davies [Dav2] and they appeared to be essentially optimal. But Davies [Dav3] has recently suggested that the bounds should be determined by two scales, the first determining the initial, or small time distribution, and the second the asymptotic, or large time distribution. In this picture the currently known bounds would give an accurate assessment of the initial heat flow but not the asymptotic flow. Davies justified his ideas by demonstrating that onedimensional systems with periodic coefficients do exhibit this multi-scale behaviour and conjectured that it is characteristic of general periodic systems. In this paper we examine the asymptotic behaviour of heat flow on stratified Lie groups with coefficients invariant under a lattice subgroup and establish that Davies' conjecture is indeed valid. But first, to fix ideas, we outline the main results of our analysis in the simple case of periodic operators acting on $\mathbf{R}^{d}$.

Let $C=\left(c_{i j}\right)$ be a real symmetric $d \times d$-matrix with components $c_{i j} \in L_{\infty}\left(\mathbf{R}^{d} ; d x\right)$ satisfying bounds $\beta_{1} I \leq C \leq \beta_{2} I$ with $\beta_{1}>0$ and let $H$ be the positive self-adjoint operator associated with the closed quadratic form

$$
\varphi \in L_{2 ; 1} \rightarrow h(\varphi)=\sum_{i, j=1}^{d}\left(\partial_{i} \varphi, c_{i j} \partial_{j} \varphi\right)
$$

where $L_{2 ; 1}$ denotes the once-differentiable functions in $L_{2}\left(=L_{2}\left(\mathbf{R}^{d} ; d x\right)\right)$. Then $H$ generates a positive self-adjoint contraction semigroup $S$ on $L_{2}$ which extends from $L_{2} \cap L_{p}$ to a contraction semigroup on $L_{p}$, also denoted by $S$, for each $p \in[1, \infty]$. The action of $S$ is determined by a positive semigroup kernel $t>0 \rightarrow K_{t} \in C_{0}\left(\mathrm{R}^{d} \times \mathrm{R}^{d}\right)$,

$$
\left(S_{t} \varphi\right)(x)=\int_{\mathbf{R}^{d}} d y K_{t}(x ; y) \varphi(y),
$$

and $K$ satisfies the Gaussian upper bound [Dav2]

$$
0 \leq K_{t}(x ; y) \leq a t^{-d / 2} e^{-d_{c}(x ; y)^{2} / 4(1+\delta) t}
$$

for all $\delta, t>0$ where $d_{c}$ denotes the geodesic distance

$$
d_{c}(x ; y)=\sup \left\{\psi(x)-\psi(y): \psi \in C_{c}^{\infty}\left(\mathbf{R}^{d}\right), \sum_{i, j=1}^{d} c_{i j} \partial_{i} \psi \partial_{j} \psi \leq 1\right\}
$$

associated with the Riemannian metric $C^{-1}$. If the $c_{i j}$ are constant one readily establishes that $d_{c}(x ; y)^{2}=\left((x-y), C^{-1}(x-y)\right)$. Then explicit calculation shows that

$$
K_{t}(x ; y)=(4 \pi t)^{-d / 2}|\operatorname{det} C|^{-1 / 2} e^{-d_{c}(x ; y)^{2} / 4 t}
$$

and for this reason the upper bounds (1) seem essentially optimal. But if the $c_{i j}$ are periodic the behaviour of $S$ for large $t$ is related to the limit as $\varepsilon \rightarrow 0$ of the semigroup 
$S^{\varepsilon}$ generated by the operator $H_{\varepsilon}$ obtained by replacing $C$ with $C^{\varepsilon}$ in the definition of $H$ where $c_{i j}^{\varepsilon}(x)=c_{i j}(x / \varepsilon)$ (see, for example, [BLP] or [ZKON]). It follows, however, from [BLP] Chapter I, that $H_{\varepsilon}$ converges in the strong resolvent sense on $L_{2}$ to an operator $\widehat{H}$ with constant coefficients $\widehat{C}=\left(\widehat{c}_{i j}\right)$. We will establish that if $\widehat{S}$ is the semigroup generated by $\widehat{H}$ then on each of the $L_{p}$-spaces, $p \in[1, \infty]$, one has the uniform convergence

$$
\lim _{t \rightarrow \infty}\left\|S_{t}-\widehat{S}_{t}\right\|_{p \rightarrow p}=0
$$

Therefore $\widehat{S}$ describes the asymptotic heat flow and the Gaussian distribution is determined by the distance $d_{\hat{c}}$ and not the original geodesic distance, which in principle could be smaller. We will also establish uniform convergence properties of $K_{t}$ and identify the limits in terms of the constant coefficient kernel $\widehat{K}_{t}$ and hence lend further justification to the two-scale picture of the heat flow.

\section{$2 \quad$ Stratified Lie groups and nilmanifolds}

We begin by recalling some elementary definitions and facts about stratified Lie groups from $[\mathrm{FoS}]$. A real Lie algebra $\mathfrak{g}$ is called stratified if it has a vector space decomposition

$$
\mathfrak{g}=\oplus_{k=1}^{\infty} \mathfrak{g}^{(k)}
$$

where all but a finite number of the subspaces $\mathfrak{g}^{(k)}$ are nonzero,

$$
\left[\mathfrak{g}^{(k)}, \mathfrak{g}^{(l)}\right] \subseteq \mathfrak{g}^{(k+l)}
$$

for all $k, l \in N$, and $\mathfrak{g}^{(1)}$ generates $\mathfrak{g}$ as a Lie algebra. Thus a stratified Lie algebra is automatically nilpotent and if $r$ is the largest integer such that $\mathfrak{g}^{(r)} \neq 0$ then $\mathfrak{g}$ is said to be nilpotent of step $r$. A Lie group is defined to be stratified if it is connected and simply
connected and its Lie algebra $\mathfrak{g}$ is stratified.

Let $G$ be a stratified Lie group and exp: $\mathfrak{g} \rightarrow G$ the exponential map. The CampbellBaker-Hausdorff formula establishes that

$$
\exp (X) \exp (Y)=\exp (H(X, Y))
$$

where $H(X, Y)=X+Y+[X, Y] / 2+$ a finite linear combination of higher order commutators in $X$ and $Y$. Thus $X, Y \rightarrow H(X, Y)$ defines a group multiplication law on the underlying vector space $V$ of $\mathfrak{g}$ which makes $V$ a Lie group whose Lie algebra is $\mathfrak{g}$ and the exponential map exp: $\mathfrak{g} \rightarrow V$ is simply the identity. Then $V$ with the group law is diffeomorphic to $G$. Next let $d_{k}$ denote the dimension of $g^{(k)}$ and $d$ the dimension of $\mathfrak{g}$ and for each $k$ choose a vector space basis $a^{(k)}=\left(a_{1}^{(k)}, \ldots, a_{d_{k}}^{(k)}\right)$ of $\mathfrak{g}^{(k)}$ such that $a_{1}, \ldots, a_{d}=a_{1}^{(1)}, \ldots, a_{d_{r}}^{(r)}$ is a basis of $\mathfrak{g}$. If $\xi_{1}, \ldots, \xi_{d}$ is the dual basis for $\mathfrak{g}^{*}$, i.e., if $\xi_{k}\left(a_{l}\right)=\delta_{k, l}$, define $\eta_{k}=\xi_{k} \circ \exp ^{-1}$. Then $\eta_{1}, \ldots, \eta_{d}$ are a system of global coordinates for
$G$ and the product rule on $G$ becomes

$$
\eta_{k}(x y)=\eta_{k}(x)+\eta_{k}(y)+P_{k}(x, y)
$$


where $P_{k}(x, y)$ is a finite sum of monomials in $\eta_{i}(x), \eta_{i}(y)$ for $i<k$ with degree between 2 and $m$. It follows that both left and right Haar measure on $G$ can be identified with Lebesgue measure $d \eta_{1} \ldots d \eta_{d}$.

If $1 \leq j \leq d_{1}$ we will need the functions $y_{j}$ on $G$ defined by

$$
y_{j}\left(\exp \left(\sum_{k=1}^{d} \eta_{k} a_{k}\right)\right)=\eta_{j}
$$

These functions satisfy the equations

$$
-A_{i}^{(l)} y_{j}=A_{i}^{(r)} y_{j}=\delta_{i j}
$$

for $1 \leq i, j \leq d_{1}$ where $A^{(l)}$ and $A^{(r)}$ denote the left and right derivatives in the direction $a \in \mathfrak{g}$. In fact these equations together with the initial conditions $y_{k}(e)=0$ determine the $y_{j}$ uniquely since all higher order commutators in the first $d_{1}$ derivatives applied to $y_{j}$ must necessarily vanish. The differential equations for the $y_{j}$ are a consequence of the Campbell-Baker-Hausdorff formula. One has

$$
\exp \left(-\varepsilon a_{i}\right) \exp \left(\sum_{k=1}^{d} \eta_{k} a_{k}\right)=\exp \left(-\varepsilon a_{i}+\sum_{k=1}^{d} \eta_{k} a_{k}+R\right)
$$

where $R$ is an $\varepsilon$-dependent element in $[\mathfrak{g}, \mathfrak{g}]$. Thus

$$
y_{j}\left(\exp \left(-\varepsilon a_{i}\right) \exp \left(\sum_{k=1}^{d} \eta_{k} a_{k}\right)\right)=\eta_{j}-\varepsilon \delta_{i j}
$$

and the differential equations follow immediately. Note that at this point it is important that $a_{1}, \ldots, a_{d_{1}}$ is a basis of $\mathfrak{g}^{(1)}$ and $\mathfrak{g}^{(1)}$ generates $\mathfrak{g}$ as a Lie algebra; the system (2) does not have a solution when $i>d_{1}$.

Next let $\Gamma$ be a subgroup of $G$ which is a lattice, i.e., $\Gamma$ is closed and discrete and $G / \Gamma$ is compact. (Since $G$ is nilpotent this is equivalent to the requirement that $G / \Gamma$ has a $G$-invariant probability measure [Hel].) Then there exists a compact subset $Y$ of $G$ such that

1. $\quad \bigcup_{\gamma \in \Gamma} Y \gamma=G$

2. $\mu\left(Y \gamma_{1} \cap Y \gamma_{2}\right)=0$ for all $\gamma_{1} \neq \gamma_{2}$ in $\Gamma$

where $\mu$ denotes Haar measure (see, for example, [Leh] pages 28-32). It is then clear that $Y$ contains at least one element from each residue class in $G / \Gamma$ and generically, i.e., on a set of full measure, only one. (One may, for example, define $Y$ by choosing a Riemannian metric $|\cdot|$ and then setting $Y$ to be the set of points $x \in G$ such that $|x|$ is smaller or equal to $|x \gamma|$ for all $\gamma \in \Gamma$. It follows that $Y$ is closed and contains at least one representative from each residue class in $G / \Gamma$. In fact $Y=\cap_{\gamma \in \Gamma} H_{\gamma}$, where $H_{\gamma}$ is the half space

$$
H_{\gamma}=\left\{x \in G:|x| \leq\left|x \gamma^{-1}\right|\right\} \text {, }
$$


and the assertions concerning $Y$ follow immediately.) The homogeneous space $G / \Gamma$ is homeomorphic to $Y / \sim$ where $\sim$ is the equivalence relation of belonging to the same residue class. phism transport the Haar measure, restricted to $Y$, to $G / \Gamma$ by means of this homeomorthen have the measure $m$ on $G / \Gamma$ which is invariant under left translations by $G$. We measure $m$ on $G / \Gamma$,

which is valid for all $f \in L_{1}(G ; d \mu)$.

$$
\int_{G} d x f(x)=\int_{G / \Gamma} d m(x) \sum_{\gamma \in \Gamma} f(x \gamma)
$$

One can define a one-parameter group of automorphisms of $G$ by first introducing the automorphisms

$$
\sum_{k=1}^{r} \sum_{l=1}^{d_{k}} \eta_{k, l} a_{l}^{(k)} \rightarrow \sum_{k=1}^{r} \sum_{l=1}^{d_{k}} \varepsilon^{k} \eta_{k, l} a_{l}^{(k)}
$$

of the Lie algebra $\mathfrak{g}$ for $\varepsilon>0$. These automorphisms then define automorphisms of $G$ which we denote by $x \in G \rightarrow \varepsilon x$. Clearly $\varepsilon_{1}\left(\varepsilon_{2} x\right)=\left(\varepsilon_{1} \varepsilon_{2}\right) x$ and if $D=\sum_{k=1}^{r} k d_{k}$ it follows from the identification of Haar measure with Lebesgue measure that $\mu(\varepsilon U)=\varepsilon^{D} \mu(U)$ for all measurable sets $U \subseteq G$. The map $x \rightarrow \varepsilon x$ is referred to as a scaling by $\varepsilon$. Now note that if $f$ is a continuous function with compact support then

$$
\int_{G} d x f(x)=m(G / \Gamma) \lim _{\varepsilon \rightarrow 0} \varepsilon^{D} \sum_{\gamma \in \Gamma} f(y \varepsilon(\gamma))
$$

where the limit is uniform in $y \in G$. This follows from the computation

$$
\int_{G} d x f(x)=\sum_{\gamma \in \Gamma} \int_{\varepsilon(Y \gamma)} d x f(x),
$$

the fact that $\mu(\varepsilon(Y \gamma))=\mu(\varepsilon Y)=\varepsilon^{D} \mu(Y)=\varepsilon^{D} m(G / \Gamma)$ and the uniform continuity of $f$. As a corollary we deduce that if $c$ is an $L_{\infty}$-function on $G$ which is periodic with periods
in $\Gamma$, i.e., $c(x \gamma)=c(x)$ for all $x \in G$ and $\gamma \in \Gamma$, and if $f \in L_{1}$ then

In the last expression we have identified $c$ with a function on $G / \Gamma$ and the relation is

$$
\lim _{\varepsilon \rightarrow 0} \int_{G} d x c(x / \varepsilon) f(x)=m(G / \Gamma)^{-1} \int_{G / \Gamma} d m(x) c(x) \int_{G} d x f(x) .
$$
established by making a change of variables,

$$
\int_{G} d x c(x / \varepsilon) f(x)=\varepsilon^{D} \int_{G} d x c(x) f(\varepsilon x)=\sum_{\gamma \in \Gamma} \varepsilon^{D} \int_{Y_{\gamma}} d x c(x) f(\varepsilon x) .
$$
If $f$ is continuous with compact support then $f(\varepsilon x)$ is close to the constant value $f(\varepsilon \gamma)$
over $Y \gamma$ and therefore

$$
\begin{aligned}
\lim _{\varepsilon \rightarrow 0} \int_{G} d x c(x / \varepsilon) f(x) & =\lim _{\varepsilon \rightarrow 0} \sum_{\gamma \in \Gamma} \varepsilon^{D} \int_{Y \gamma} d x c(x) f(\varepsilon \gamma) \\
& =\lim _{\varepsilon \rightarrow 0} \sum_{\gamma \in \Gamma} \varepsilon^{D} \int_{Y} d x c(x) f(\varepsilon \gamma)
\end{aligned}
$$




$$
\begin{aligned}
& =\int_{Y} d x c(x) \lim _{\varepsilon \rightarrow 0} \sum_{\gamma \in \Gamma} \varepsilon^{D} f(\varepsilon \gamma) \\
& =\int_{Y} d x c(x) m(G / \Gamma)^{-1} \int_{G} d x f(x) \\
& =m(G / \Gamma)^{-1} \int_{G / \Gamma} d m(x) c(x) \int_{G} d x f(x)
\end{aligned}
$$

by the previous observation. If $f$ is only an $L_{1}$-function the result follows by approximating $f$ in the $L_{1}$-norm with continuous functions of compact support.

\section{Operator theory}

In this section $G$ is a stratified group with Lie algebra $\mathfrak{g}$ and we adopt the notation of the previous section. Now for each $a \in \mathfrak{g}$ and $f \in C^{\infty}(G)$ we define the left derivative

$$
(A f)(x)=\left.\frac{d}{d t} f(\exp (-t a) x)\right|_{t=0}
$$

for all $x \in G$. Thus if $L$ denotes the left regular representation of $G$ one has $A=d L(a)$. Since $d L(a)$ commutes with right translations it passes to the quotient $M=G / \Gamma$, i.e., if $f$ is $\Gamma$-periodic then $A f$ is also $\Gamma$-periodic. But since $M$ carries an invariant measure $m$ the representation $L$ induces a unitary representation $V$ of $G$ on $L_{2}(M ; m)$ given by

$$
(V(g) f)(x \Gamma)=f\left(g^{-1} x \Gamma\right)
$$

for all $x, g \in G$ and $f \in L_{2}(M ; m)$. It is convenient to denote the Lie isomorphism $a \rightarrow d V(a)$ by $a \rightarrow B$ to distinguish it from $a \rightarrow A=d L(a)$.

Let $C=\left(c_{i j}\right)$ be a $d_{1} \times d_{1}$-matrix with coefficients $c_{i j} \in L_{\infty}(G ; d x)$ satisfying ellipticity bounds $\beta_{1} I \leq C \leq \beta_{2} I$, with $\beta_{1}>0$, uniformly over $G$. Then there is a unique self-adjoint operator on $L_{2}(G ; d x)$ canonically associated with the closed quadratic form

$$
h(f)=\sum_{i, j=1}^{d_{1}}\left(A_{i} f, c_{i j} A_{j} f\right)
$$

with domain $D(h)=L_{2 ; 1}^{\prime}$ where

$$
L_{2 ; 1}^{\prime}(G ; d x)=\bigcap_{i=1}^{d_{1}} D\left(A_{i}\right) .
$$

and it is conventional to write

$$
H=-\sum_{i, j=1}^{d_{1}} A_{i} c_{i j} A_{j}
$$

The self-adjoint contraction semigroup $S$ generated by $H$ leaves $L_{2} \cap L_{p}$ invariant for each $p \in[1, \infty]$ and extends to a contraction semigroup, also denoted by $S$, on each of the 
spaces $L_{p}=L_{p}(G ; d x)$. Recall that the $a_{i}$ in (4) are a vector space basis of $\mathfrak{g}^{(1)}$ and hence an algebraic basis of $\mathfrak{g}$ by the stratification assumption. Thus $H$ is a subelliptic operator operator on $L_{2}(M ; m)$ and the semigroup $S$. periodic then $H$ can also be viewed as an

We adopt the methods used in the spaces $L_{p}(M ; m)$. as described in [BLP] and consider the operators

$$
\widetilde{H}_{\varepsilon}=-\sum_{i, j=1}^{d_{1}}\left(A_{i}+\varepsilon^{-1} B_{i}\right) c_{i j}(y)\left(A_{j}+\varepsilon^{-1} B_{j}\right),
$$

where $\varepsilon>0$ and $B_{i}=d V\left(a_{i}\right)$, acting on functions $u$ on $G \times M$, i.e., functions $u$ on $G \times G$ which satisfy $u(x, y \gamma)=u(x, y)$ for all $x, y \in G$ and $\gamma \in \Gamma$. Next consider the equation

$$
\lambda u_{\varepsilon}+\widetilde{H}_{\varepsilon} u_{\varepsilon}=f
$$

where $\lambda \in \mathbf{C}$ with $\operatorname{Re} \lambda \geq 0$, and where $u_{\varepsilon}$ has the formal expansion

$$
u_{\varepsilon}=u_{0}+\varepsilon u_{1}+\varepsilon^{2} u_{2}+\ldots
$$

and $f \in L_{2}$. Note that if $H_{\varepsilon}$ is the operator obtained by replacing the coefficients $c_{i j}$ of $H$ by the rescaled coefficients $c_{i j}^{\varepsilon}$ where $c_{i j}^{\varepsilon}(x)=c_{i j}\left(\varepsilon^{-1} x\right)$ then $(6)$ is equivalent to the
equation

$$
H_{\varepsilon} v_{\varepsilon}+\lambda v_{\varepsilon}=f
$$

with $v_{\varepsilon}(x)=u_{\varepsilon}\left(x, \varepsilon^{-1} x\right)$. It is this observation which motivates the introduction of $\widetilde{H}_{\varepsilon}$.

The lowest order terms in $\varepsilon$ of the equation (6) give the system of equations

where

$$
\begin{gathered}
H_{2} u_{0}=0, \\
H_{2} u_{1}+H_{1} u_{0}=0, \\
H_{2} u_{2}+H_{1} u_{1}+H_{0} u_{0}=f,
\end{gathered}
$$

$$
\begin{gathered}
H_{0}=-\sum_{i, j=1}^{d_{1}} c_{i j}(y) A_{i} A_{j}+\lambda I \\
H_{1}=-\sum_{i, j=1}^{d_{1}}\left(B_{i} c_{i j}(y) A_{j}+A_{i} c_{i j}(y) B_{j}\right) \\
H_{2}=-\sum_{i, j=1}^{d_{1}} B_{i} c_{i j}(y) B_{j}
\end{gathered}
$$

The solution of this system of equations involves the homogenized operator defined by

$$
\widehat{H}=-\sum_{i, j=1}^{d_{1}} \widehat{c}_{i j} A_{i} A_{j}
$$


where the constant coefficients $\hat{c}_{i j}$ are given by

$$
\widehat{c}_{i j}=\int_{M} d m\left(c_{i j}-\sum_{k=1}^{d_{1}} c_{i k} B_{k} f_{j}\right)
$$

and the functions $f_{j}$ are weak solutions of the differential equations

$$
H_{2} f_{j}=-\sum_{i=1}^{d_{1}} B_{i} c_{i j}
$$

Noting that $\mathrm{H}_{2}$ is the self-adjoint operator associated with the form $h$ restricted to the domain

$$
L_{2 ; 1}^{\prime}=\bigcap_{i=1}^{d_{1}} D\left(B_{i}\right)
$$

one has $D\left(B_{i}\right) \subseteq D\left(H_{2}^{1 / 2}\right) \subseteq D\left(H_{2}\right)$. Hence the differential equations (9) are soluble if and only if there exist $f_{j} \in L_{2}(M ; m)$ such that

$$
\left(H_{2} f, f_{j}\right)=\sum_{i=1}^{d_{1}}\left(B_{i} f, c_{i j}\right)
$$

for all $f \in D\left(H_{2}\right)$. But $L_{2}(M ; m)$ has a $V$-invariant direct sum decomposition $\mathcal{H}_{\mathbf{R}} \oplus \mathcal{H}_{\mathrm{R}}^{\perp}$ where $\mathcal{H}_{\mathbf{R}}$ is the one-dimensional subspace spanned by the constant functions and $\mathcal{H}_{\mathbf{R}}$ its orthogonal complement. Now the value of $f_{j}$ restricted to $\mathcal{H}_{\mathbf{R}}$ is arbitrary. and we choose it to be zero. Then the problem of constructing the $f_{j}$ reduces to finding $\tilde{f}_{j} \in \mathcal{H}_{\mathrm{R}}^{\frac{1}{\mathrm{R}}}$ such that

$$
\left(H_{2} f, \tilde{f}_{j}\right)=\sum_{i=1}^{d_{1}}\left(B_{i} f, \tilde{c}_{i j}\right)
$$

for all $f \in D\left(H_{2}\right) \cap \mathcal{H}_{\mathrm{R}}^{\perp}$ where

$$
\tilde{c}_{i j}=c_{i j}-\int_{M} d m(y) c_{i j}(y)
$$

Next remark that the ellipticity condition on the $c_{i j}$ gives

$$
\beta_{1} \Delta \leq H_{2} \leq \beta_{2} \Delta
$$

where $\Delta$ is the positive self-adjoint sublaplacian

$$
\Delta=-\sum_{i=1}^{d_{1}} B_{i}^{2} .
$$

But on $\mathcal{H}_{\mathrm{R}}^{\frac{1}{\mathrm{f}}}$ the sublaplacian is strictly positive. Thus one has bounds

$$
H_{2} \geq \beta_{1} \Delta \geq \beta_{1} \omega I
$$

with $\omega>0$. Hence $H_{2}^{-1}$ is bounded on $\mathcal{H}_{\mathbf{R}}^{\frac{1}{R}}$ and in particular $\left\|H_{2}^{-1 / 2}\right\| \leq\left(\beta_{1} \omega\right)^{-1 / 2}$. Moreover,

$$
\beta_{1}\left\|B_{i} f\right\|_{2}^{2} \leq\left\|H_{2}^{1 / 2} f\right\|_{2}^{2}
$$


for all $f \in L_{2 ; 1}^{\prime}(M ; m)=D\left(H_{2}^{1 / 2}\right)$. Hence $\left\|B_{i} H_{2}^{-1 / 2}\right\| \leq \beta_{1}^{-1 / 2}$ for all $i=1, \ldots, d_{1}$. Combining these estimates one concludes that the operators $X_{i}=B_{i} H_{2}^{-1}$ are bounded on $\mathcal{H}_{\mathrm{R}}^{\perp}$ and $\left\|X_{i}\right\| \leq \beta^{-1} \omega^{-1 / 2}$. Therefore the $\tilde{f}_{j}$ are uniquely determined by

$$
\tilde{f}_{j}=\sum_{i=1}^{d_{1}} X_{i}^{*} \tilde{c}_{i j}
$$

and $f_{j}=0 \oplus \tilde{f}_{j}$ and it follows from this construction that $\tilde{f}_{j} \in L_{2 ; 1}^{\prime}(M ; m)$.

The coefficients of the homogenized operator can also be computed with the aid of the coordinates $y_{i}, i=1, \ldots, d_{1}$, introduced in Section 2. One has the representation

$$
\begin{aligned}
\widehat{c}_{i j} & =\int_{Y} d y \sum_{k, l=1}^{d_{1}} A_{k}\left(f_{i}(y)-y_{i}\right) c_{k l}(y) A_{l}\left(f_{j}(y)-y_{j}\right) \\
& =h_{2}\left(f_{i}-y_{i}, f_{j}-y_{j}\right)
\end{aligned}
$$

where $h_{2}$ denotes the sesquilinear form associated with $H_{2}$. This can be verified from (8) and (9) by repetition of the argument of [BLP], pages 17-18. Note that this representation establishes that $\widehat{C}$ is positive-definite. One can in fact strengthen this conclusion. The homogenized matrix satisfies the bounds

$$
\beta_{1} I \leq \widehat{C} \leq \beta_{2} I
$$

where the $\beta_{i}$ are the ellipticity constants of $C$. These bounds follow by the reasoning on pages $31-35$ of [BLP] which again involves the coordinates $y_{i}$. In this proof and at various later stages of the argument compactness arguments are used. The basic observation is that if $\Omega$ and $\Omega^{\prime}$ are open, precompact, sets in $G$ with $\bar{\Omega} \subseteq \Omega^{\prime}$ then the restriction map $L_{2 ; 1}^{\prime}\left(\Omega^{\prime} ; d x\right) \rightarrow L_{2}(\Omega ; d x)$ is compact. This is established by first noting that if $\varphi$ is in the subset of $L_{2 ; 1}^{\prime}\left(\Omega^{\prime} ; d x\right)$ with $\|\varphi\|_{2 ; 1, \Omega^{\prime}} \leq 1$, then $\|\varphi\|_{2} \leq 1$, i.e., the set is bounded in

$$
\|L(x) \varphi-\varphi\|_{2, \Omega} \leq c|x|, \quad\|R(x) \varphi-\varphi\|_{2, \Omega} \leq c|x|
$$

for $x \in G$ sufficiently close to the identity $e$ that $x^{-1} \bar{\Omega} \subseteq \Omega^{\prime}$ and $\bar{\Omega} x \subseteq \Omega^{\prime}$. Here $L$ and $R$ denote left and right translations and $|x|=d_{c}(x ; e)$. The compactness then follows from the Fréchet-Kolmogorov criterion (see, for example, [Rob] Appendix D). If one restricts attention to $\Omega$ with smooth boundaries then one can establish somewhat more; the restriction map $L_{2 ; 1}^{\prime}(\Omega ; d x) \rightarrow L_{2}(\Omega ; d x)$ is compact. The first compactness statement is equivalent to the observation that the Dirichlet sublaplacian on $L_{2 ; 1}^{\prime}\left(\Omega^{\prime} ; d x\right)$ has compact resolvent and the second is equivalent to compactness of the resolvent of the Neumann
sublaplacian on $L_{2 ; 1}^{\prime}(\Omega ; d x)$.

The identification (10) can also be expressed as a variational principle. Let $g_{1}, \ldots, g_{d_{1}} \in$ $L_{2 ; 1}^{\prime}(M ; m)$ and consider the associated positive-definite matrix $C(g)$ with components
defined by

$$
c_{i j}(g)=h_{2}\left(g_{i}-y_{i}, g_{j}-y_{j}\right) \text {. }
$$


Then $\widehat{C}=C(f)$. More generally one has $C(g) \geq \widehat{C}$ with equality if and only if $g_{i}=f_{i}$, up to the addition of a constant, for all $i=1, \ldots, \bar{d}_{1}$.

If $G=\mathbf{R}$ then there is only one coefficient $c$ and the corresponding constant $\hat{c}$ can be computed as

$$
\widehat{c}=\left(p^{-1} \int_{0}^{p} d x c(x)^{-1}\right)^{-1}
$$

where $p$ now denotes the period of $c$. This is discussed on pages 8-9 of [BLP] and is used by Davies [Dav3] in his analysis of one-dimensional systems. tion.

The significance of the homogenized operator $\widehat{H}$ is explained by the following proposi-

Proposition 3.1 Let $H_{\varepsilon}, \varepsilon>0$, denote the subelliptic operators obtained by replacing the coefficients $c_{i j}$ of $H$ by $c_{i j}^{\varepsilon}$ where $c_{i j}^{\varepsilon}(x)=c_{i j}\left(\varepsilon^{-1} x\right)$. For $\lambda \in \mathrm{C}$ with $\operatorname{Re} \lambda>0$ consider the equations

where $f \in L_{2}(G ; d x)$.

$$
\lambda u_{\varepsilon}+H_{\varepsilon} u_{\varepsilon}=f
$$

It follows that these equations have unique solutions $u_{\varepsilon} \in L_{2}(G ; d x)$. As $\varepsilon \rightarrow 0$ the $u_{\varepsilon}$ converge strongly in $L_{2, \mathrm{loc}}$ and their derivatives $A_{i} u_{\varepsilon}, i=1, \ldots, d_{1}$, converge weakly in $L_{2, \text { loc }}$. The limit $u_{0}$ of the $u_{\varepsilon}$ is the unique solution of the equation

$$
\lambda u_{0}+\widehat{H} u_{0}=f
$$

where $\widehat{H}$ is the homogenized operator given by (7).

The proposition is very similar to Theorem 3.1 in [BLP] and its proof is almost identical. The theorem of [BLP] concerns Dirichlet operators on bounded regions in $\mathbf{R}^{d}$ but the proof is based on general reasoning which extends to the Lie group setting. The fact that $L_{2 ; 1}^{\prime}(\Omega ; d x)$ is compactly embedded in $L_{2}(\Omega ; d x)$ for each bounded open subset $\Omega$ of $G$ is again important and the coordinates $y_{i}$ introduced in Section 2 are also essential for the argument. These coordinates replace the polynomial $P$ used in the $[B L P]$ proof. A minor new element is the introduction of the term $\lambda$ in the differential equation but this alone does not significantly alter the reasoning in [BLP].

The local convergence $u_{\varepsilon} \rightarrow u_{0}$ given by Proposition 3.1 can be reexpressed in terms of the resolvents $\left(\lambda I+H_{\varepsilon}\right)^{-1}$ and $(\lambda I+\widehat{H})^{-1}$. Observing that $u_{\varepsilon}=\left(\lambda I+H_{\varepsilon}\right)^{-1} f$ one has

$$
\lim _{\varepsilon \rightarrow 0}\left(\varphi,\left(\left(\lambda I+H_{\varepsilon}\right)^{-1}-(\lambda I+\widehat{H})^{-1}\right) f\right)=0
$$

for all $f \in L_{2}(G ; d x), \varphi \in C_{c}(G)$ and all $\lambda \in \mathrm{C}$ with $\operatorname{Re} \lambda \geq 0$. Approximating elements of $L_{2}$ by $\varphi \in C_{c}$ and using uniform norm bounds on the resolvents it is evident that one
has weak resolvent convergence, i.e.,

$$
\lim _{\varepsilon \rightarrow 0}\left(g,\left(\left(\lambda I+H_{\varepsilon}\right)^{-1}-(\lambda I+\widehat{H})^{-1}\right) f\right)=0
$$


for all $f, g \in L_{2}(G ; d x)$ and all $\lambda \in \mathbf{C}$ with $\operatorname{Re} \lambda \geq 0$. But this implies strong resolvent
convergence by use of the resolvent identity

$$
\left(\bar{\lambda} I+H_{\varepsilon}\right)^{-1}\left(\lambda I+H_{\varepsilon}\right)^{-1}=(2 i \operatorname{Im} \lambda)^{-1}\left(\left(\bar{\lambda} I+H_{\varepsilon}\right)^{-1}-\left(\lambda I+H_{\varepsilon}\right)^{-1}\right)
$$

and a similar identity for the resolvent of $\widehat{H}$.

Corollary 3.2 Adopt the assumptions of Proposition 3.1 and let $S^{\varepsilon}$ and $\hat{S}$ denote the self-adjoint contraction semigroups on $L_{2}(G ; d x)$ generated by $H_{\varepsilon}$ and $\widehat{H}$, respectively.

It follows that

for all $f \in L_{2}(G ; d x)$ and $t>0$.

$$
\lim _{\varepsilon \rightarrow 0}\left\|\left(S_{t}^{\varepsilon}-\widehat{S}_{t}\right) f\right\|_{2}=0
$$

The strong convergence of the semigroups is a standard consequence of the strong bounds and regeresolvents. In the next section we will demonstrate that by use of kernel bouncis and regularity properties the convergence can be improved to uniform convergence.

\section{Asymptotic properties}

The asymptotic properties of $S_{t}$ as $t \rightarrow \infty$, where $S$ is the semigroup generated by the operator $H$ with $\Gamma$-periodic coefficients, are closely related to the convergence properties $S$ is determined by a positive kernel $K$
of the semigroups $S^{\varepsilon}$ generated by the rescaled $H_{\varepsilon}$. The action of the semigroup

which now satisfies Gaussian bounds

$$
\left(S_{t} f\right)(x)=\int_{G} d y K_{t}(x ; y) f(y)
$$

$$
0 \leq K_{t}(x ; y) \leq a t^{-D / 2} e^{-b d_{c}(x ; y)^{2} / t}
$$

for all $\delta, t>0$ where the dimension $D$ is defined in Section 2 and $d_{c}$ denotes the geodesic
distance

$$
d_{c}(x ; y)=\sup \left\{\psi(x)-\psi(y): \psi \in C_{c}^{\infty}(G), \sum_{i, j=1}^{d_{1}} c_{i j} A_{i} \psi A_{j} \psi \leq 1\right\} .
$$

The value of the parameters $a, b$ in (12) only depend on the coefficients $c_{i j}$ of $H$ through the ellipticity constants $\beta_{1}, \beta_{2}$. Thus if $K^{\varepsilon}$ denotes the kernels associated with the semigroups $S^{\varepsilon}$ obtained by rescaling then these satisfy the same Gaussian bounds, i.e., the bounds are uniform in $\varepsilon$. Moreover, the $K^{\varepsilon}$ satisfy the scaling relations

$$
K_{t}^{\varepsilon}(x ; y)=\varepsilon^{-D} K_{\varepsilon^{-2} t}\left(\varepsilon^{-1} x ; \varepsilon^{-1} y\right)
$$
for all $\varepsilon, t>0$ and $x, y \in G$. The kernel $\widehat{K}$ of the homogenized semigroup $\widehat{S}$ generated by
the constant coefficient operator $\widehat{H}$ satisfies bounds similar to (12) but with the distance
corresponding to the coefficients $\widehat{c}_{i j}$. It also satisfies the corresponding to the coefficients $\widehat{c}_{i j}$. It also satisfies the scaling relation

$$
\widehat{K}_{t}(x ; y)=\varepsilon^{-D} \widehat{K}_{\varepsilon^{-2} t}\left(\varepsilon^{-1} x ; \varepsilon^{-1} y\right)
$$


for all $\varepsilon, t>0$ and $x, y \in G$.

Although there is an extensive literature on Gaussian bounds the bounds we have cited for operators with measurable coefficients do not seem to appear in the literature. They follow, however, by the refinement of Nash's arguments [Nas] given by Fabes and Stroock $[\mathrm{FaS}]$ and one can in fact show that one may choose $b=1 / 4(1+\delta)$ for any $\delta>0$ but the value of $a$ then depends upon the choice of $\delta$. The important point is that all the estimates used by Nash are quadratic form estimates. (A detailed exposition of the Nash-FabesStroock method for the case of constant coefficients can be found in [Rob], Chapter IV.) Saloff-Coste and Stroock [SCS] have given Gaussian bounds for smooth coefficients but they observe that the smoothness hypothesis is not essential.

In the following statement of the asymptotic results we use $\|X\|_{p \rightarrow r}$ to denote the norm of an operator $X$ from $L_{p}$ to $L_{r}$ and in addition we define

$$
\||f|\|_{p}=\operatorname{ess~sup}_{x \in G}\left(\int_{G} d y|f(x ; y)|^{p}\right)^{1 / p}
$$

for $p \in[1, \infty)$ and

$$
\left|\|f\|_{\infty}=\underset{x, y \in G}{\operatorname{ess} \sup }\right| f(x ; y) \mid
$$

for each measurable $f: G \times G \rightarrow \mathbf{R}$.

Theorem 4.1 Let $S$ denote the semigroup generated by the subelliptic operator $H$ with $\Gamma$-periodic, $L_{\infty}$-coefficients $C=\left(c_{i j}\right)$ and let $K$ denote the corresponding kernel. Further let $\widehat{S}$ and $\widehat{K}$ denote the semigroup and kernel corresponding to the homogenized operator $\widehat{H}$ defined by (7) and (8).

Then for $1 \leq p \leq r \leq \infty$

$$
\lim _{t \rightarrow \infty} t^{D / 2 \delta}\left\|S_{t}-\widehat{S}_{t}\right\|_{p \rightarrow r}=0
$$

where $\delta=1 / p-1 / r$. Moreover,

$$
\lim _{t \rightarrow \infty} t^{D / 2 q}\left|\left\|K_{t}-\widehat{K}_{t} \mid\right\|_{p}=0\right.
$$

where $1 / p+1 / q=1$.

Proof The proof is based on first establishing convergence properties of the kernels and then converting these into convergence properties of the semigroups by observing that

$$
\left\|S_{t}-\widehat{S}_{t}\right\|_{p \rightarrow \infty}=\left\|\mid K_{t}-\widehat{K}_{t}\right\|_{q}
$$

with $1 / p+1 / q=1$. Initially we obtain a local convergence of the kernels.

Proposition 4.2 Adopt the assumptions of Theorem 4.1. Then

$$
\lim _{t \rightarrow \infty} \operatorname{ess}_{|x|^{2}+|y|^{2} \leq a t}\left|K_{t}(x ; y)-\widehat{K}_{t}(x ; y)\right|
$$

for each $a>0$ where $|x|=d_{c}(x, e)$. 
Proof In the case $G=\mathbf{R}^{d}$ this follows immediately from Corollary 3.2 together with Theorem 4 of [ZKON]. For a general stratified Lie group $G$ the arguments of [ZKON] are still applicable since $G$ is a polynomial group and Saloff-Coste and Stroock [SCS] have extended Nash's [Nas] Hölder regularity estimates to this case. It then follows that the family of kernels $\left\{K_{1}^{\varepsilon}: \varepsilon>0\right\}$ is equicontinuous and bounded so Ascoli's theorem shows that the family is relatively compact in the topology of uniform convergence on compacts. (In [SCS] the coefficients are assumed to be smooth but the estimates do not depend upon the derivatives and it is observed that smoothness is not essential. The regularity follows from upper and lower Gaussian bounds and Nash's method of deriving these, as elaborated in $[\mathrm{FaS}]$ relies only on quadratic form techniques.)

Since the scaling relations (13) and (14) are valid the remaining arguments of [ZKON] are unchanged.

Next the local convergence is extended to global convergence of the kernels.

Proposition 4.3 Adopt the assumptions of Theorem 4.1. Then

$$
\lim _{t \rightarrow \infty} t^{D / 2}\left\|\left|K_{t}-\widehat{K}\right|\right\|\left\|_{\infty}=0 \quad, \quad \lim _{t \rightarrow \infty}\right\| \mid K_{t}-\widehat{K} \|_{1}=0
$$

Proof Since $K$ satisfies the Gaussian bounds (12) and $\widehat{K}$ satisfies similar bounds one can choose for each $\delta>0$ an $a>0$ such that

$$
\sup _{\left|x^{-1} y\right|^{2}>a t} t^{D / 2}\left|K_{t}(x ; y)-\widehat{K}_{t}(x ; y)\right|<\delta
$$

and

$$
\int_{\left|x^{-1} y\right|^{2}>a t} d y\left|K_{t}(x ; y)-\widehat{K}_{t}(x ; y)\right|<\delta
$$

for all $x \in G$. The second estimate follows from the fact that the ball of radius $\lambda$ in $G$ is proportional to $\lambda^{D}$ by scaling. But it follows from Proposition 4.2 that

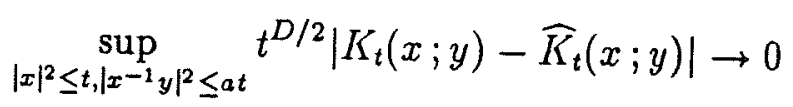

and

$$
\sup _{|x|^{2} \leq t} \int_{\left|x^{-1} y\right|^{2} \leq a t} d y\left|K_{t}(x ; y)-\widehat{K}_{t}(x ; y)\right| \rightarrow 0
$$

as $t \rightarrow \infty$. It follows from (15) and (17) that

$$
\sup _{|x|^{2} \leq t, y \in G} t^{D / 2}\left|K_{t}(x ; y)-\widehat{K}_{t}(x ; y)\right| \rightarrow 0
$$

and from (16) and (18) that

$$
\sup _{|x|^{2} \leq t} \int_{G} d y\left|K_{t}(x ; y)-\widehat{K_{t}}(x ; y)\right| \rightarrow 0
$$

as $t \rightarrow \infty$. 
Since the coefficients $c_{i j}$ of $H$ are $\Gamma$-periodic it follows that

$$
K_{t}(x \gamma ; y \gamma)=K_{t}(x ; y)
$$

for all $x, y \in G$, all $\gamma \in \Gamma$ and all $t>0$. Moreover,

$$
\widehat{K}_{t}(x z ; y z)=\widehat{K}_{t}(x ; y)
$$

for all $x, y, z \in G$ and $t>0$. But as $\Gamma$ is a lattice there exists $\tau>0$ such that each coset in $G / \Gamma$ contains a point $x$ such that $|x|^{2} \leq \tau$. It then follows from (19), (21) and (22) that

$$
\begin{aligned}
t^{D / 2}||\left|K_{t}-\widehat{K}_{t}\right| \|_{\infty} & =\sup _{|x|^{2} \leq \tau, \gamma \in \Gamma, y \in G} t^{D / 2}\left|K_{t}(x \gamma ; y)-\widehat{K}_{t}(x \gamma ; y)\right| \\
& =\sup _{|x|^{2} \leq \tau, \gamma \in \Gamma, y \in G} t^{D / 2}\left|K_{t}\left(x ; y \gamma^{-1}\right)-\widehat{K}_{t}\left(x ; y \gamma^{-1}\right)\right| \rightarrow 0
\end{aligned}
$$

as $t \rightarrow \infty$. Similarly it follows from (20), (21) and (22) that

$$
\begin{aligned}
\left\|K_{t}-\widehat{K}_{t}\right\| \|_{1} & =\sup _{|x|^{2} \leq \tau, \gamma \in \Gamma} \int_{G} d y\left|K_{t}(x \gamma ; y)-\widehat{K}_{t}(x \gamma ; y)\right| \\
& =\sup _{|x|^{2} \leq \tau, \gamma \in \Gamma} \int_{G} d y\left|K_{t}(x \gamma ; y \gamma)-\widehat{K}_{t}(x \gamma ; y \gamma)\right| \\
& =\sup _{|x|^{2} \leq \tau} \int_{G} d y\left|K_{t}(x ; y)-\widehat{K}_{t}(x ; y)\right| \rightarrow 0
\end{aligned}
$$

as $t \rightarrow \infty$.

Now we can complete the proof of Theorem 4.1 .

The convergence statement for the kernels follows for $p=1$ and $p=\infty$ from Proposition 4.2 and then for $p \in\langle 1, \infty\rangle$ by interpolation. Then

$$
t^{D / 2 p}\left\|S_{t}-\widehat{S}_{t}\right\|_{p \rightarrow \infty}=t^{D / 2 p}\|\| K_{t}-\widehat{K}_{t} \|_{q}
$$

and so the statement on semigroup convergence follows for $r=\infty$. But

$$
\left\|S_{t}-\widehat{S}_{t}\right\|_{1 \rightarrow 1}=\left\|S_{t}-\widehat{S}_{t}\right\|_{\infty \rightarrow \infty}=\left\|\mid K_{t}-\widehat{K}_{t}\right\|_{1}
$$

and so the case $p=r=1$ is also established. The general result then follows by interpolation.

\section{Conclusion}

The conclusion

$$
\lim _{t \rightarrow \infty} t^{D / 2}\left\|K_{t}-\widehat{K}_{t}\right\|_{\infty}=0
$$

given by Theorem 4.1 demonstrates clearly that the Gaussian spread of the distribution $K$ is asymptotically described by the Gaussian associated with the constant coefficient 
operator $\widehat{H}$. In the simplest case $G=\mathbf{R}$ this result was obtained by Davies [Dav3] who
also proved that $\left\|\mid K_{t}-\widehat{K}_{t}\right\|_{\infty} \leq c t^{-1}$. It appears reasonable to

$$
t^{D / 2}\left\|\mid K_{t}-\widehat{K}_{t}\right\|_{\infty} \leq c t^{-1 / 2}
$$

at least for large values of $t$. This would correspond to estimates

$$
\left\|K_{t}^{\varepsilon}-\widehat{K}_{t}\right\|_{\infty} \leq c \varepsilon
$$

for fixed $t$ and small $\varepsilon$, on the rescaled kernels and these estimates are indicated by the results of [BLP].

Davies also demonstrated in the one-dimensional case that

$$
\begin{aligned}
\lim _{\varepsilon \rightarrow 0} d_{c^{c}}(x ; y) & =\lim _{\varepsilon \rightarrow 0} \varepsilon^{2} d_{c}\left(\varepsilon^{-1} x ; \varepsilon^{-1} y\right) \\
& \leq d_{\hat{c}}(x ; y)
\end{aligned}
$$

with equality if and only if the coefficient $c$ is constant. This inequality then allows one to $\widehat{K}$, which that the asymptotic bounds on the heat kernel which follow from the bounds on relies upon the explicit ide $d_{\hat{c}}$, improve the earlier bounds in terms of $d_{c}$. Davies proof operator. In higher dimensions, form of $\hat{C}$ but it is again reasonable to expect that $G$, it is more difficult to exploit the

$$
\lim _{\varepsilon \rightarrow 0} d_{c^{e}}(x ; y) \leq d_{\hat{c}}(x ; y)
$$

with equality only in the case of constant coefficients. This corresponds to an upper semicontinuity of the distance as a function of the coefficients.

\section{References}

[BLP]

$$
\begin{aligned}
& \text { Bensoussan, A., Lions, J.L. And PAPANICOLAOU, G.C., Asymptotic anal- } \\
& \text { ysis for periodic structures. Studies in Mathematics and its Applications } 5 . \\
& \text { North-Holland, } 1978 .
\end{aligned}
$$

[Dav1] Davies, E.B., Heat kernels and spectral theory. Cambridge Tracts in Mathe-
matics 92. Cambridge University Press, Cambridge etc., 1989.

matics 92. Cambridge University Press, Cambridge etc., 1989.

[Dav3]

Amer. Journ. Math. 109 (1987), 319-333.

$[\mathrm{FaS}]$ versity, 1991.

FABES, E.B. and STroock, D.W., A new proof of Moser's parabolic Harnack 327-338. 
[FoS] Folland, G.B. and Stein, E.M., Hardy spaces on homogeneous groups. Mathematical Notes 28. Princeton University Press, Princeton, 1982.

[Hel] Helgason, S., Differential geometry, Lie groups and symmetric spaces. Academic Press, New York, 1978.

[Leh] LEhNER, J., A short course in automorphic functions. R. W. Holt Publishing Inc., New York, 1966.

[Nas] NASH, J., Continuity of solutions of parabolic and elliptic equations. Amer. J. Math. 80 (1958), 931-954.

[Rob] RobInson, D.W., Elliptic operators and Lie groups. Oxford Mathematical Monographs. Oxford University Press, Oxford etc., 1991.

[SCS] Saloff-Coste, L. and STroock, D.W., Opérateurs uniformement souselliptiques sur les groupes de Lie. J. Funct. Anal. 98 (1991), 97-121.

[VSC] Varopoulos, N., Saloff-Coste, L. and Coulhon, T., Analyse sur les groupes de Lie. Research report, Université Paris VI, 1989.

[ZKON] Zhikov, V.V., Kozlov, S.M., Oleinik, O.A. and Kha T'EN NgoAN, Averaging and $G$-convergence of differential operators. Russ. Math. Surveys 34 (1979), 69-147. 